\title{
Single Phase Sine Wave PWM Inverter Circuit Simulation And The Design Of Filter Based On Matlab
}

\author{
Niu Yanshu ${ }^{1, \mathrm{a}}$ \\ ${ }^{1}$ School of Electrical and Electronic Engineering, North China Electric Power University, Changping District, Beijing 102206, China
}

\begin{abstract}
Inverter circuit is the most important application of PWM control technology. This paper mainly discusses the unipolar PWM ( pulse width modulation ) control mode of single-phase bridge inverter circuit, and uses MATLAB visual simulation tool Simulink to model the circuit. At the same time, a filter is designed to filter the waveform of the inverted output so that the output waveform can reach a smooth sine wave shape. This simulation not only deepens the understanding of PWM inverter circuit theory, but also lay a good experimental foundation for the study of modern power electronics.
\end{abstract}

\section{Introduction}

In power electronics technology, converting direct current into alternating current is called inversion. Inverter circuits are widely used, for example, inverter circuits are needed when DC power supplies AC loads. Inverter circuit is also the core part of power electronic devices such as frequency converter for AC motor speed regulation, uninterruptible power supply and induction heating power supply[1].

At present, the inverter technology mainly uses pulse width modulation ( PWM ). Because the characteristics of PWM wave determine that the output voltage of the power amplifier device contains more harmonic components[2], filters must be used in the inverter output to make the output waveform reach a smooth sine wave shape and reduce the influence of harmonics on the load.

\section{Simulation of single-phase sine wave PWM inverter circuit}

The simulation model of single-phase sine wave inverter circuit modulated by unipolar PWM is shown in figure 1 . the DC power supply voltage in the main circuit is $200 \mathrm{v}$, which is connected to a resistive inductive load via a bridge inverter circuit formed by universal - bridge module[3]. The load inductance is $2 \mathrm{mh}$ and the resistance is $3 \Omega$.

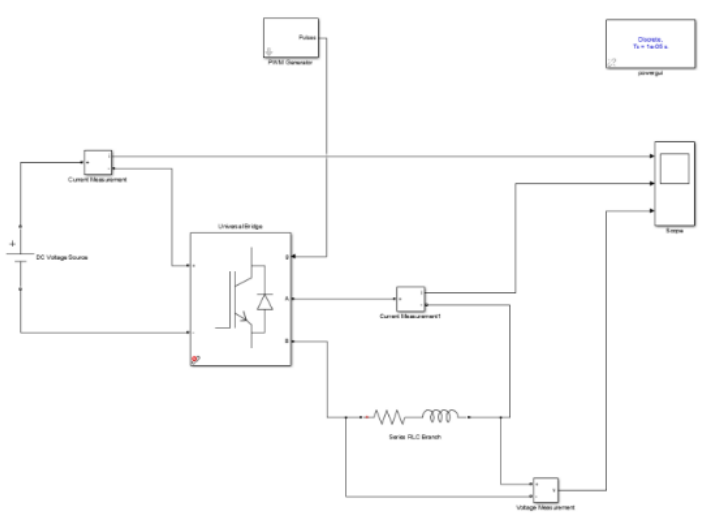

Figure 1. Simulation model of single-phase sinusoidal PWM inverter.

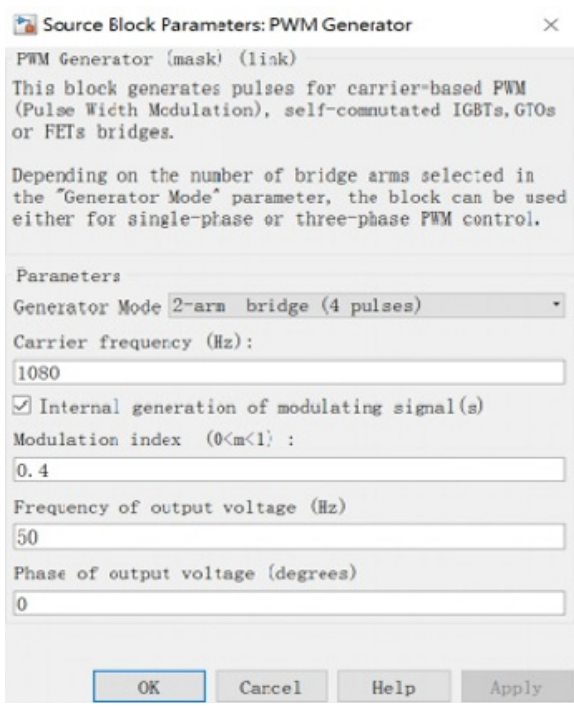

Figure 2. PWM generator parameter setting.

\footnotetext{
a Corresponding author: yanshu_niu@163.com
} 


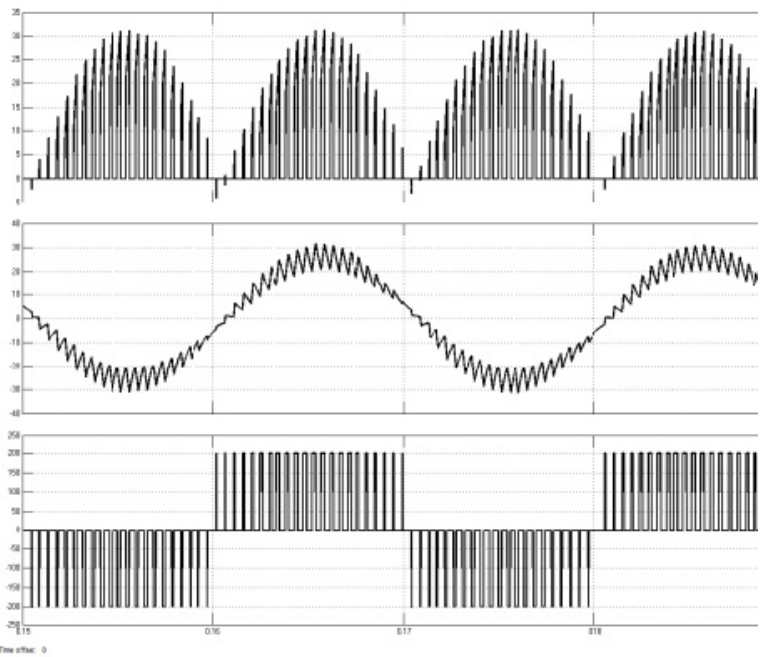

Figure 3. Simulation waveform of single-phase sinusoidal PWM inverter circuit.

The parameter settings of universal - bridge module are shown in figure 2 . The simulation waveform of the circuit is shown in figure 3. It can be seen from the waveform that the result is completely consistent with the real experiment result, which proves the correctness of the simulation result.

\section{Design of filter}

\subsection{Parameter setting of filter}

When designing filters, the value of inductance is usually designed first. The selection of inductance is related to the ripple current and the power consumption of the system. In general, the ripple current on the inductor 1 is selected to be $15 \%-25 \%$ of the rated current. In the case of unipolar modulation [4]:

$$
\Delta I_{1}=\frac{U_{\mathrm{d}}-U_{o}(t)}{L} \times \frac{D(t)}{f_{e}}
$$

Under the condition that the switching frequency is far greater than the working frequency, according to the principle of unipolar PWM, the duty ratio of each switching period can be obtained as follows

$$
D(\mathrm{t})=\frac{U_{\mathrm{o}}(\mathrm{t})}{U_{\mathrm{d}}}
$$

It can be obtained by substituting equation (2) into equation (1)

$$
\Delta I_{1}=\frac{U_{\mathrm{d}}-U_{\mathrm{o}}(\mathrm{t})}{L} \times \frac{U_{\mathrm{o}}(\mathrm{t})}{f_{e} \times U_{d}}
$$

$$
\begin{aligned}
& \text { As can be seen from equation } \\
& U_{\mathrm{o}}(\mathrm{t})=\frac{U_{\mathrm{d}}}{2} \text {, there is a maximum value }
\end{aligned}
$$
when

$$
\Delta I_{1 \max }=\frac{U_{\mathrm{d}}}{4 L f_{c}}
$$

So there is

$$
L \geq \frac{U_{\mathrm{d}}}{4 f_{c} \Delta I_{l \max }}
$$

The cut-off frequency of LC filter $f_{c}$ is

$$
f_{c}=\frac{1}{2 \pi \sqrt{L C}}
$$

$$
\text { among them } 10 f \leq f_{c} \leq f_{\text {har }(\min ) / 2}
$$

In the formula : $f$ the fundamental frequency; $f_{\text {har (min) }}$ is the lowest harmonic frequency.

If the current ripple of the filter inductor is approximately regarded as a sine wave and it is assumed that all of them are absorbed by the filter capacitor, then the maximum value of the output voltage ripple should be

$$
\Delta U_{\text {omax }}=\Delta I_{\operatorname{lmax}} \times \frac{1}{2 \pi f_{c} C}
$$
(7)

Formula (4) can be obtained by substituting formula

$$
\Delta U_{\text {omax }}=\frac{U_{\mathrm{d}}}{8 \pi f_{c}^{2} L C}
$$

Obtain following equation

$$
L C \geq \frac{U_{\mathrm{d}}}{8 \pi f_{c}^{2} \Delta U_{o \text { max }}}
$$

The value of $\mathrm{L}$ can be obtained from equation (5) and the value of $\mathrm{C}$ can be calculated from equation (6). The parameter settings for $\mathrm{L}$ are shown in figure 4 and the parameter settings for $\mathrm{C}$ are shown in figure 5 .

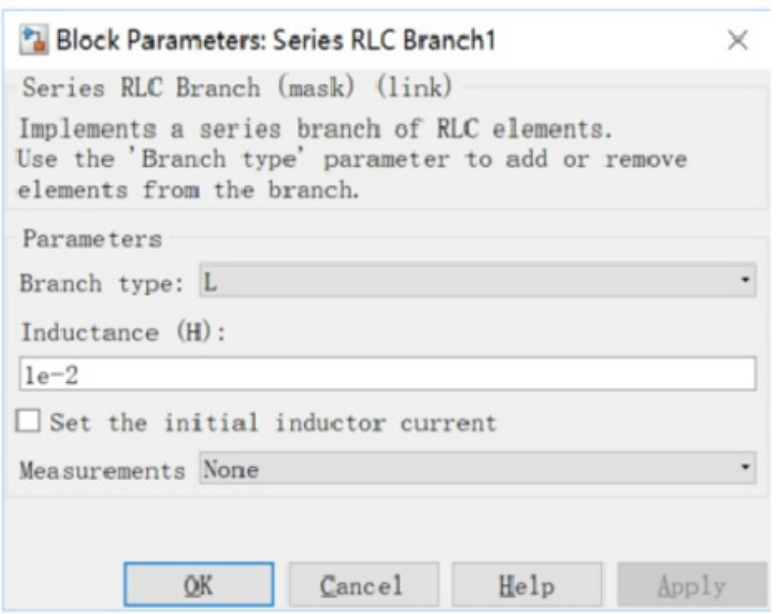

Figure 4. Parameter setting of inductance in filter. 


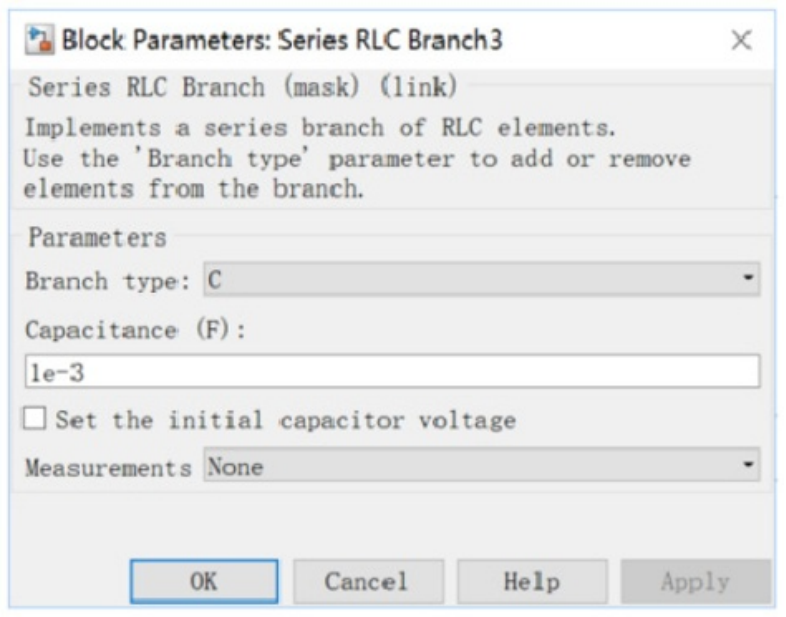

Figure 5. Parameter setting of capacitance in filter.

\subsection{Establishment of simulation model}

The simulation circuit of LC filter is shown in figure 6, and the output waveform is shown in figure 7 .

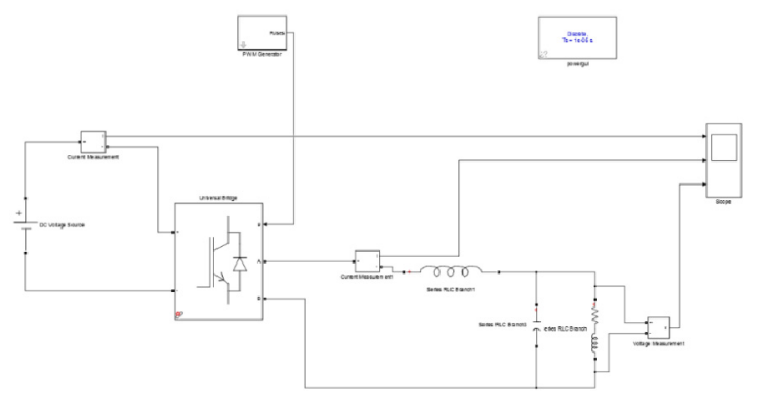

Figure 6. Simulation circuit with LC filter.
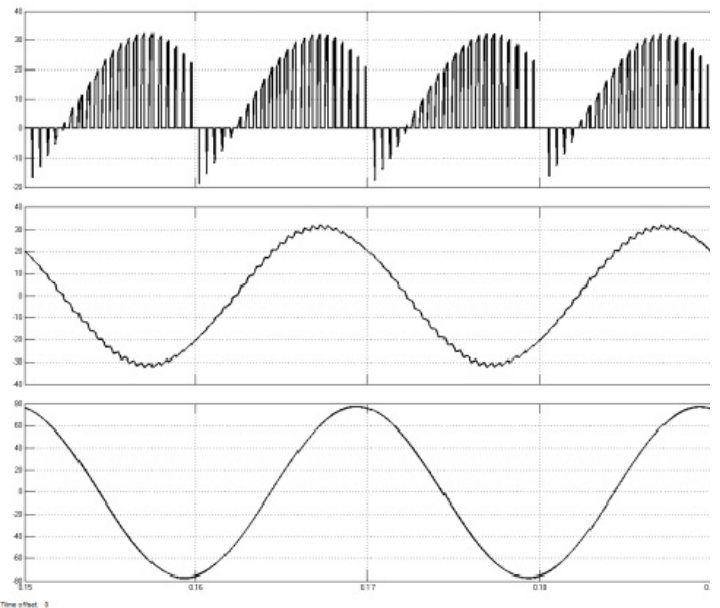

Figure 7. Output waveform of circuit after adding LC filter.

Through the LC filter designed by the above method, the simulation experiment shows that the high frequency components can be filtered out and the relatively smooth sine wave can be output by further adjusting the parameters of $\mathrm{L}$ and $\mathrm{C}$.

\section{Harmonic analysis}

The harmonic analysis of the output current obtained by the single-phase sinusoidal PWM inverter circuit without filter is carried out through the " power GUI". the result is shown in figure 8 . THD $=13.62 \%$, which is much larger than the $8 \%$ content of the national standard[5].

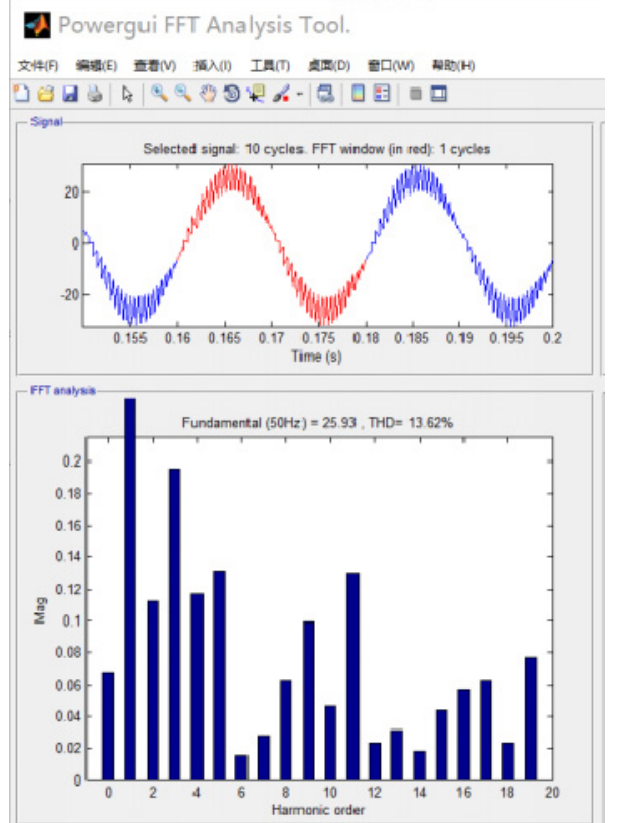

Figure 8. Harmonic analysis of output current without filter.

The output current obtained by the single-phase sinusoidal PWM inverter circuit is filtered by a filter and analyzed by " power GUI". the result is shown in figure 9. $\mathrm{THD}=2.30 \%$, which greatly reduces the harmonic content and meets the national standard.

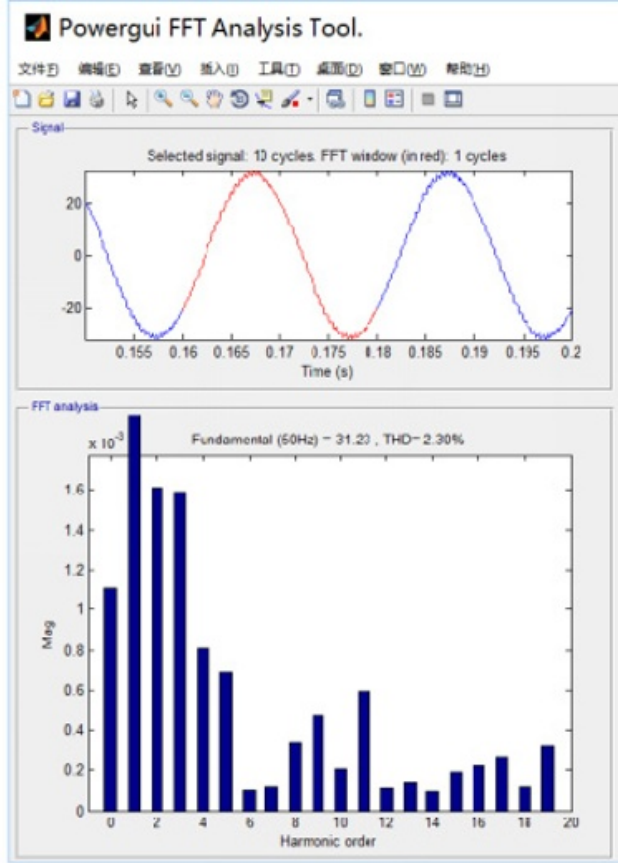

Figure 9. Harmonic analysis of output current with filter.

\section{Concluding remarks}

In order to make the PWM inverter have a better output waveform, the second order LC low pass filter network [6] is used in view of the high harmonic frequency of the PWM inverter. 
From the simulation results, it can be seen that after LC filtering is added, the harmonic components in the output voltage are filtered out, which is closer to the sine wave shape, while the waveform of the load current is smoother and the sine degree is better. It shows that adding filter link can obviously improve the inverter output waveform.

\section{References}

1. Nan Zhu, Libing Zhang, Weichuan Ye, Junpei Xu.Simulation research on single phase PWM inverter circuit based on MATLAB[J].Electronics World,2012(07):70-72.

2. Xianwen Wei.Simulation research on PWM inverter circuit based on MATLAB[J].Electronic Technology \& Software Engineering,2014(11):9091.

3. Dalei $\mathrm{Wu}$, Simulation of sine wave inverter circuit based on MATLAB / Simulink[J].China CIO News, 2010(06):144+36.

4. Yingguang He, Xiaohong Ren, Gang Fang, Tianwen Wang.Optimal design of output filter for singlephase PWM inverter[J].Electric Drive,2010,40(11): 33-35.

5. Dayi Li, Qiaofu Chen, Zhengchun Jia. Power harmonic analysis method based on FFT function in MATLAB $[\mathrm{J}]$. Electrical Measurement \& Instrumentation, 2002(07):23-26.

6. Yangwei $\mathrm{Yu}$, Tianjun Jin, Wentao Xie, Zhengyu Lv.Lc filter based on PWM inverterJ].Mechanical \& Electrical Engineering Magazine,2007(05):50-52. 